\title{
Bariatric Surgery During the COVID-19 Pandemic - the Perspective of Physicians and Patients
}

\author{
Atefeh Fakharian $^{1} \cdot$ Hedieh Amin Moghadassi ${ }^{1} \cdot$ Maryam Vasheghani $^{1,2}\left(\mathbb{D} \cdot\right.$ AliAkbar Amin Moghadasi $^{3}$
}

Received: 16 August 2020 / Revised: 16 September 2020 / Accepted: 17 September 2020 / Published online: 22 September 2020

(C) Springer Science+Business Media, LLC, part of Springer Nature 2020

In December 2019, a new virus from the coronavirus strain has caused pneumonia in patients in Wuhan, China. The World Health Organization (WHO) named the virus COVID-19, which rapidly became pandemic. According to the WHO report on September 2, 2020, the total confirmed COVID-19 cases were $25,602,665$, of whom 852,758 have died. Mortality from COVID-19 is higher than that from influenza A, severe acute respiratory syndrome-coronavirus (SARS-CoV), and Middle East respiratory syndrome (MERS) [1, 2].

Currently, no specific treatment or approved effective vaccine is available, so sanitation, social distancing, quarantine, and control of COVID-19 risk factors are the most effective way to control the disease $[3,4]$.

Both COVID-19 and obesity are pandemic [5] and study of obesity impact on COVID-19 disease could be an interesting topic. According to available data and clinical practice at the US Centers for Disease Control and Prevention [6], morbid obesity (body mass index or BMI $\geq 40 \mathrm{~kg} / \mathrm{m}^{2}$ ) is a risk factor for poor prognosis and higher mortality in patients with

Maryam Vasheghani

mvasheghani9@gmail.com

Atefeh Fakharian

fakharian_2005@yahoo.com

Hedieh Amin Moghadassi

hedieh.moghadassi@yahoo.com

AliAkbar Amin Moghadasi

aminmoghadasi_2008@yahoo.com

1 Chronic Respiratory Diseases Research Center (CRDRC), National Research Institute of Tuberculosis and Lung Diseases (NRITLD), Shahid Beheshti University of Medical Sciences, Tehran, Iran

2 Internal Medicine Department, Masih Daneshvari Hospital, Daraabad Avenue, Shahid Bahonar roundabout, Post Box: 19575154, Tehran 1956944413, Iran

3 Surgery Ward, Toos General Hospital, Tehran, Iran
COVID-19. In addition, any degree of obesity (BMI $\geq$ $30 \mathrm{~kg} / \mathrm{m}^{2}$ ) is associated with worse prognosis in patients with COVID-19. Therefore, obesity increases the incidence, severity, and duration of COVID-19.

The association between obesity and COVID-19 can be assessed in several aspects. First, obesity causes respiratory system dysfunctions such as decreased chest wall elasticity, lung compliance, and expiratory reserve volume [7]. Second, obesity is associated with disorders such as type 2 diabetes mellitus, atherosclerosis, hypertension and cardiovascular diseases, and cancer [8] that have also been identified as risk factors for COVID-19. These comorbidities may exacerbate COVID-19 and increase the likelihood of hospitalization in the intensive care unit (ICU) and mortality [9]. Third, there is a hypercoagulopathy state and hyperinflammation in obesity. Inflammatory markers such as interleukin-6 and C-reactive protein are increased in obese individuals $[10,11]$. Hypercoagulation and the increase of inflammatory markers such as interleukin-6 have been seen in COVID-19, which are sometimes so severe that they cause clots in vital organs and cytokine storm syndrome [12]. In these conditions, the disease is severe and the risk of admission to the ICU and mortality is high. Whether obesity exacerbates COVID-19 by increasing inflammatory factors or hypercoagulation, or it is just a coincidence, is still unclear and requires further research.

On the other hand, COVID-19 also has an effect on obesity. COVID-19 increases social constraints and reduces mobility, and increases the weight and prevalence of obesity in society, and this vicious cycle continues.

Obesity treatments such as diet and physical activity are often effective in the short term. Bariatric surgery (BS) is one of the effective treatments for obesity in the long term [13]. The health care system has been facing one of the most important dilemmas to treat obesity.

There is a risk of infection transmission during patient admission and metabolic surgery, and the signs and symptoms of the disease make it difficult for patients to be followed up after surgery [14]. A cohort study was 
performed in Italy on patients with and without COVID19 who were candidates for various surgeries. In patients with COVID-19, the risks of 30-day mortality and surgical, pulmonary, and thrombotic complications were 9.5, $4.98,35.6$, and 13.2 times higher than those without COVID-19, respectively. Therefore, due to the COVID19 pandemic and the rapid disease spread, some pulmonologists and bariatric surgeons believe that BS is an elective operation and it is better to postpone surgery [15].

Others believe that obesity is one of the risk factors for admission to the ICU and COVID-19 severity and mortality, so it is not an elective surgery [16] and postponing metabolic surgery due to the complications of morbid obesity, comorbidities such as diabetes, the role of obesity, and these comorbidities on COVID-19 may increase risk of death and complications [17]. According to the American Society for Metabolic and Bariatric Surgery (ASMBS), "The BS is not an elective surgery and the lives of patients with obesity are in danger and the bariatric surgery is much safer than the COVID-19 patients with obesity" [18].

At present, there is insufficient data on the advantages and complications of bariatric surgery during the COVID-19 pandemic, and different scientific societies have different recommendations, so the views of patients and physicians also play a role in deciding on bariatric surgery.

According to a study in Argentina [19], in which 83 surgeons have been asked to fill in a questionnaire about how they passed the coronavirus pandemic condition, almost all preferred virtual consultation while these services are free of charge in $48 \%$ of cases. Based on the above study, $90 \%$ of them prefer laparoscopic surgery over other surgical methods. During surgery, even if the patient is not suspected of having a viral infection, in $80,56.6$, and $66 \%$ of cases, they tend to use an N95 mask, face shield, and smoke filter for pneumo-peritoneum, respectively. Only $10.8 \%$ would perform surgery as usual with routine protective measures. To resume elective surgery, $56.6 \%$ believe that the hospital should be in Yellow Code (Phase 0 or I; Phase 0: Warning, Phase 1: Emergency Department resources have been exhausted and are in need of additional resources) [20], the patient should be carefully selected according to scientific criteria, and the scientific associations of surgeons should approve the resumption of this type of surgery. The patient selection criteria with the most consensuses are as follows: test to rule out asymptomatic patients with positive COVID-19 test, negative history of chronic lung disease, age $<60$ years, and healthy immune system. Because of the risk of infection, about one-fifth of them consider sleeve gastrectomy to be the method of choice, but the rest do not change their surgical procedure [19].
According to an online survey of obese patients before and after bariatric surgery (group I and group II) in Poland, about three-quarters of them were concerned about their health during the COVID-19 pandemic. Most of them (72\%) considered obesity to be a risk factor for COVID-19, which complicates the course of the disease. One-third of them had changed their routine diet and gained weight during the pandemic. Threefifths of them had decreased physical activity. Half of the patients, despite the risk of COVID-19, agreed to undergo surgery and $42 \%$ prefer a face-to-face visit. Almost all patients considered remote consultation with a nutritionist and psychologist, and participation in support groups to be useful and effective in continuing treatment. Most of them have access to their surgeon and treatment team during the COVID-19 pandemic, but only half of them used it. In group I, diet change, weight gain, and desire for surgery were more than those in group II. More than $87 \%$ of them wanted to have bariatric surgery at the same center after the COVID-19 pandemic, and only $2.5 \%$ decided to change the type of surgery. In response to what stage should obesity surgery be resumed, $28 \%$ believed that when new cases of COVID-19 start to decline, $11 \%$ when the vaccine is available, $47 \%$ when the WHO announces the end of the pandemic, and $14 . \%$ when the last patient is discharged from the hospital. Two-thirds believed that priority was given to patients whose surgery was delayed due to a pandemic. Seventeen percent of them believed that their health was endangered by limitations of obesity care and postponing the surgery. Virtual consult and telemedicine with the specialists of the Obesity Treatment Center can help patients to continue their scheduled weight loss [21].

In conclusion, considering the risks and complications of morbid obesity and surgery during the COVID-19 pandemic, it is reasonable to postpone metabolic surgery in patients with COVID-19 disease. Virtual consult and telemedicine may be suitable to continue weight loss programs. BS may be a great help to reduce the risk of COVID-19, severity of disease, ICU admission, and mortality in high-risk patients with morbid obesity and concomitant diseases, with careful and scientific selection of patients and strict observance of health protocols and preventive measures. There is a need for designing and conducting further studies in this field in order to make a logical and correct decision based on evidence. Of course, the patient ultimately chooses the time and type of treatment.

\section{Compliance with Ethical Standards}

Conflict of Interest The authors declare that they have no conflict of interest.

Ethical Approval This article does not contain any studies with human participants or animals performed by any of the authors.

Informed Consent Informed consent does not apply. 


\section{References}

1. Guessoum SB, Lachal J, Radjack R, et al. Adolescent psychiatric disorders during the COVID-19 pandemic and lockdown. Psychiatry Res. 2020 Sep;291:113264. https://doi.org/10.1016/j. psychres.2020.113264.

2. WHO Coronavirus Disease (COVID-19) Dashboard- overview, data last updated: 2020/9/2, 3:56pm CEST. 2020. https:// COVID19.who.int. Accessed 2 Sept 2020.

3. Vijayvargiya P, Esquer Garrigos Z, Castillo Almeida NE, et al. Treatment considerations for COVID-19: a critical review of the evidence (or lack thereof). Mayo Clin Proc. 2020;95(7):1454-66. https://doi.org/10.1016/j.mayocp.2020.04.027.

4. Kaur SP, Gupta V. COVID-19 vaccine: a comprehensive status report. Virus Res. 2020;288:198114. https://doi.org/10.1016/j. virusres.2020.198114.

5. Ritter A, Kreis NN, Louwen F, et al. Obesity and COVID-19: molecular mechanisms linking both pandemics. Int J Mol Sci. 2020;21(16):E5793. https://doi.org/10.3390/ijms21165793.

6. Sanchis-Gomar F, Lavie CJ, Mehra MR, et al. Obesity and outcomes in COVID-19: when an epidemic and pandemic collide. Mayo Clin Proc. 2020;95(7):1445-53. https://doi.org/10.1016/j. mayocp.2020.05.006.

7. Uccelli M, Cesana GC, Ciccarese F, et al. COVID-19 and obesity: postoperative risk in patients who have undergone bariatric surgery. Preliminary report from high volume center in Italy (Lombardy). Obes Surg. 2020;27:1-4. https://doi.org/10.1007/s11695-02004792-x.

8. Blüher M. Obesity: global epidemiology and pathogenesis. Nat Rev Endocrinol. 2019;15:288-98. https://doi.org/10.1038/s41574-0190176-8.

9. Banerjee M, Gupta S, Sharma P, et al. Obesity and COVID-19: a fatal alliance. Indian J Clin Biochem. 2020:1-8. https://doi.org/10. 1007/s12291-020-00909-2.

10. Chiappetta S, Sharma AM, Bottino V, et al. COVID-19 and the role of chronic inflammation in patients with obesity. Int J Obes. 2020;44(8):1790-2. https://doi.org/10.1038/s41366-020-0597-4.

11. Pasquarelli-do-Nascimento G, Braz-de-Melo HA, Faria SS, et al. Hypercoagulopathy and adipose tissue exacerbated inflammation may explain higher mortality in COVID-19 patients with obesity. Front Endocrinol (Lausanne). 2020;11:530. https://doi.org/10. 3389/fendo.2020.00530
12. Teuwen LA, Geldhof V, Pasut A, et al. COVID-19: the vasculature unleashed. Nat Rev Immunol. 2020:1-3. https://doi.org/10.1038/ s41577-020-0343-0.

13. Sudlow AC, le Roux CW, Pournaras DJ. Review of advances in anti-obesity pharmacotherapy: implications for a multimodal treatment approach with metabolic surgery. Obes Surg. 2019;29(12): 4095-104. https://doi.org/10.1007/s11695-019-04206-7.

14. Aminian A, Kermansaravi M, Azizi S, et al. Bariatric surgical practice during the initial phase of COVID-19 outbreak. Obes Surg. 2020;30(9):3624-7. https://doi.org/10.1007/s11695-020-04617-x.

15. Doglietto F, Vezzoli M, Gheza F, et al. Factors associated with surgical mortality and complications among patients with and without coronavirus disease 2019 (COVID-19) in Italy. JAMA Surg. 2020;155(8):1-14. https://doi.org/10.1001/jamasurg.2020.2713.

16. Executive Council of ASMBS. ASMBS Guidelines/Statements. Safer through surgery: American Society for Metabolic and Bariatric Surgery statement regarding metabolic and bariatric surgery during the COVID-19 pandemic. Surg Obes Relat Dis. 2020;16(8):981-2. https://doi.org/10.1016/j.soard.2020.06.003.

17. Kassir R, Rebibo L, Genser L, et al. SOFFCO-MM guidelines for the resumption of bariatric and metabolic surgery during and after the COVID-19 pandemic. J Visc Surg. 2020;157(4):317-27. https://doi.org/10.1016/j.jviscsurg.2020.06.005.

18. Beskow A, Duartez PM, Beheren E, et al. COVID-19 pandemic and bariatric surgery in Argentina. Res Square. 2020. https://www. researchsquare.com/article/rs-29759/v1.pdf. Accessed 2 Sept 2020.

19. University of Toledo. Emergency conditions \& basic staff response. https://www.utoledo.edu/depts/safety/docs/Hospital_EP/ EmergencyCodesSimplified.pdf. Accessed 2 Sept 2020.

20. Lim S, Shin SM, Nam GE, et al. Proper management of people with obesity during the COVID-19 pandemic. J Obes Metab Syndr. 2020;29(2):84-98. Published online 2020 Jun 16. https://doi.org/ 10.7570/jomes20056.

21. Walędziak M, Różańska-Walędziak A, Pędziwiatr M, et al. Bariatric surgery during COVID-19 pandemic from patients' point of view-the results of a national survey. J Clin Med. 2020;9(6): 1697. Published 2020 Jun 2. https://doi.org/10.3390/jcm9061697.

Publisher's Note Springer Nature remains neutral with regard to jurisdictional claims in published maps and institutional affiliations. 